\title{
Thermodynamics' zeroth law in a nonextensive scenario
}

\author{
S. Martínez ${ }^{\mathrm{a}, \mathrm{b}, *}$, F. Pennini ${ }^{\mathrm{a}, \mathrm{b}}$, A. Plastino ${ }^{\mathrm{a}, \mathrm{b}}$ \\ ${ }^{a}$ Departamento de Física, Facultad de Ciencias Exactas, Instituto de Física, Universidad Nacional de La \\ Plata (UNLP), C.C. 727, 1900 La Plata, Argentina \\ ${ }^{\mathrm{b}}$ Instituto de Física La Plata (IFLP), CONICET-UNLP, La Plata, Argentina
}

Received 29 September 2000; received in revised form 29 November 2000

\begin{abstract}
We show how to reconcile Tsallis' thermostatistics with thermodynamics' zeroth law, by recourse to the so-called optimal Lagrange multipliers formalism. The central concept is that of not identifying in the usual fashion the inverse temperature with the Lagrange multiplier associated to the internal energy. Our analysis provides one with compatibility conditions between the additivity of the internal energy and the pseudo-additivity of the generalized entropy. With regards to the first law of thermodynamics, a generalization of Clausius' equation is advanced. (C) 2001 Elsevier Science B.V. All rights reserved.
\end{abstract}

PACS: 05.30.-d; 05.70.Ce

Keywords: Tsallis thermostatistics; Zeroth law; First law

\section{Introduction}

Tsallis' thermostatistics allows for a suitable and quite significant generalization of the conventional (Boltzmann-Gibbs) statistical mechanics that has found multiple applications [1-14]. However, it cannot yet comfortably deal with thermodynamics' zeroth law, as pointed out by Tsallis himself in [2]. In Ref. [15] Abe has advanced

\footnotetext{
* Corresponding author. Departmento de Fisica, Facultad de Ciencias Exactas, Instituto de Fisica, Universidad Nacional de La Plata, C.C. 727, 1900 La Plata, Argentina.

E-mail addresses: martinez@venus.fisica.unlp.edu.ar (S. Martínez), pennini@venus.fisica.unlp.edu.ar (F. Pennini), plastino@venus.fisica.unlp.edu.ar (A. Plastino).
} 
an interesting, tentative solution to the zeroth law conundrum with reference to the micro-canonical analysis of a system composed of two-subsystems in thermal equilibrium. Such an analysis leads to the appearance of temperatures that depend upon the non-extensive partition function $\bar{Z}_{q}$, where $q$ is the Tsallis' non-extensivity index.

Let $\hat{\rho}$ be the density operator and $k$ the Boltzmann constant, ${ }^{1}$ or more generally, the information unit. As shown by the present authors in a recent study [16], one can recast Tsallis' variational problem (using normalized expectation values) in such a manner that the extremum one thereby finds is guaranteed to correspond to a maximum (and not to other types of extrema) of Tsallis information measure

$$
\frac{S_{q}}{k}=\frac{1-\operatorname{Tr}\left(\hat{\rho}^{q}\right)}{q-1},
$$

because the associated Hessian is diagonal. This treatment involves a new set of Lagrange multipliers $\lambda_{j}^{\prime}$, to be referred to as the "Optimal set" (OLM), different, but related, to the original Tsallis-Mendes-Plastino (TMP) one $\left(\lambda_{j}\right.$ 's) [1]

$$
\lambda_{j}^{\prime}=\frac{\lambda_{j}}{\bar{Z}_{q}^{1-q}},
$$

where the partition function $\bar{Z}_{q}$ is involved.

Another interesting work in this context is that of Ref. [17], where it is shown that for those particular systems whose partition function is given by $Z_{B G} \propto l^{a}\left(\beta^{\prime}\right)^{-a}$ ( $a$ is a dimensionless parameter, and $l$ is a characteristic length), the inverse (thermodynamical) temperature becomes associated with our $\beta^{\prime}$ and not with the TMP $\beta$.

In the present effort we tackle the vexing zeroth law problem starting with the working hypothesis that $1 / \beta^{\prime}$ is indeed the temperature (see Eq. (18) below). We show that such a hypothesis reconciles Tsallis' formalism with the zeroth law.

We shall tackle the zeroth law problem starting with the hypothesis of Ref. [15]: one deals with the Hamiltonian of a system composed of two independent subsystems (in the sense that their mutual interaction is negligible). The system's density operator is product of those pertaining to the subsystems. Before proceeding further, however, a short recapitulation is necessary.

\section{OLM formalism}

\subsection{Generalities}

The most general quantal treatment is made in a basis-independent way, which requires consideration of the statistical operator (or density operator) $\hat{\rho}$ that maximizes Tsallis' entropy, subject to the foreknowledge of $M$ generalized expectation values (corresponding to $M$ operators $\hat{O}_{j}$ ).

\footnotetext{
${ }^{1}$ Here we take $k$ to be a universal constant. The possibility of allowing $k$ to depend upon the system's nature will be explored elsewhere.
} 
Tsallis' normalized probability distribution [1] is obtained by following the well known MaxEnt route [18,19]. Instead of effecting the variational treatment of Ref. [1], involving Lagrange multipliers $\lambda_{j}$, we pursue the alternative path developed in Ref. [16], with Lagrange multipliers $\lambda_{j}^{\prime}$. One maximizes Tsallis' generalized entropy (1) $[10,11,20]$ subject to the constraints (generalized expectation values) $[10,16]$

$$
\begin{aligned}
& \operatorname{Tr}(\hat{\rho})=1, \\
& \operatorname{Tr}\left[\hat{\rho}^{q}\left(\hat{O}_{j}-\left\langle\hat{O}_{j}\right\rangle_{q}\right)\right]=0,
\end{aligned}
$$

where $\hat{O}_{j}(j=1, \ldots, M)$ denote the $M$ relevant observables (the observation level [23], whose generalized expectation values [1]

$$
\left\langle\hat{O}_{j}\right\rangle_{q}=\frac{\operatorname{Tr}\left(\hat{\rho}^{q} \hat{O}_{j}\right)}{\operatorname{Tr}\left(\hat{\rho}^{q}\right)},
$$

are (assumedly) a priori known. The resulting density operator reads [16]

$$
\hat{\rho}=\bar{Z}_{q}^{-1}\left[1-(1-q) \sum_{j}^{M} \lambda_{j}^{\prime}\left(\hat{O}_{j}-\left\langle\hat{O}_{j}\right\rangle_{q}\right)\right]^{1 / 1-q},
$$

where $\bar{Z}_{q}$ stands for the partition function

$$
\bar{Z}_{q}=\operatorname{Tr}\left[1-(1-q) \sum_{j} \lambda_{j}^{\prime}\left(\hat{O}_{j}-\left\langle\hat{O}_{j}\right\rangle_{q}\right)\right]^{1 / 1-q} .
$$

It is shown in Ref. [16] that

$$
\operatorname{Tr}\left(\hat{\rho}^{q}\right)=\bar{Z}_{q}^{1-q},
$$

and that Tsallis' entropy can be cast as

$$
S_{q}=k \ln _{q} \bar{Z}_{q}
$$

with $\ln _{q} \bar{Z}_{q}=\left(1-\bar{Z}_{q}^{1-q}\right) /(q-1)$. These results coincide with those of TMP [1] in their normalized treatment. If, following [1], we define now

$$
\ln _{q} Z_{q}=\ln _{q} \bar{Z}_{q}-\bar{Z}_{q}^{1-q} \sum_{j} \lambda_{j}^{\prime}\left\langle\hat{O}_{j}\right\rangle_{q},
$$

we are straightforwardly led to Ref. [16]

$$
\begin{aligned}
& \frac{\partial}{\partial\left\langle\hat{O}_{j}\right\rangle_{q}}\left(\frac{S_{q}}{k}\right)=\bar{Z}_{q}^{1-q} \lambda_{j}^{\prime}=\lambda_{j} \\
& \frac{\partial}{\partial \lambda_{j}^{\prime}}\left(\ln _{q} Z_{q}\right)=-\bar{Z}_{q}^{1-q}\left\langle\hat{O}_{j}\right\rangle_{q} .
\end{aligned}
$$


Eqs. (11) and (12) are modified Information Theory relations of the type that one uses to build up, à la Jaynes [18,19], Statistical Mechanics. The basic Legendre-structure relations can be recovered in the limit $q \rightarrow 1$. These relations are exactly the same as those introduced in Ref. [1] in terms of the TMP's Lagrange multipliers, but from our new point of view they lose their basic character, because the set $\lambda_{j}$ depend upon the partition function.

As a special instance of Eqs. (11) and (12) let us discuss the Canonical Ensemble, where they adopt the appearance

$$
\begin{gathered}
\frac{\partial}{\partial U_{q}}\left(\frac{S_{q}}{k}\right)=\bar{Z}_{q}^{1-q} \beta^{\prime}=\beta \\
\frac{\partial}{\partial \beta^{\prime}}\left(\ln _{q} Z_{q}\right)=-\bar{Z}_{q}^{1-q} U_{q},
\end{gathered}
$$

with (see Eq. (10))

$$
\ln _{q} Z_{q}=\ln _{q} \bar{Z}_{q}-\beta^{\prime} \bar{Z}_{q}^{1-q} U_{q} .
$$

\subsection{Legendre relations}

We present here some new results with reference to the OLM formalism of the preceding subsection. Obviously, Eqs. (11) and (12) do not have the usual appearance of Legendre relations cast in terms of the partition function because the natural logarithm is replaced by the $q$-logarithm. This difference makes the inverse $q$-temperature $\beta$, a fictitious pseudo inverse-temperature that only leads to the conventional thermodynamic one in the limit $q \rightarrow 1$.

Now, the entropy (1) can be re-expressed in terms of the partition function, by recourse to (8), in the fashion $S_{q} / k=\left(1-\bar{Z}_{q}^{1-q}\right) /(q-1)$, so that

$$
\frac{\partial}{\partial\left\langle\hat{O}_{j}\right\rangle_{q}}\left(\frac{S_{q}}{k}\right)=\bar{Z}_{q}^{-q} \frac{\partial \bar{Z}_{q}}{\partial\left\langle\hat{O}_{j}\right\rangle_{q}} .
$$

This, in turn, allows one to write (11) in the form

$$
\frac{\partial \ln \bar{Z}_{q}}{\partial\left\langle\hat{O}_{j}\right\rangle_{q}}=\lambda_{j}^{\prime},
$$

which does preserve the standard structure of a thermodynamics Legendre relation (a natural logarithm involved). Specialization to the canonical ensemble gives

$$
\frac{\partial \ln \bar{Z}_{q}}{\partial U_{q}}=\beta^{\prime},
$$

which makes the OLM formalism a bridge between Tsallis' statistics and conventional Thermodynamics. The OLM Lagrange multipliers acquire thereby the conventional thermodynamic interpretation. 
The foregoing remarks are to be kept in mind in re-discussing the zeroth law. Before addressing our main theme, the connection between the OLM formalism and the first law of thermodynamics is to be also refreshed. See the appendix.

\section{Thermodynamical equilibrium}

We tackle now the question we wish to address in this effort: to discuss anew the problem of thermodynamical equilibrium on the basis of the results of the preceding section. Let us consider a composed isolated Hamiltonian system $A+B$, within the framework of the Microcanonical Ensemble. These two subsystems interact via heat exchange.

The zeroth law asserts that if $A$ is in equilibrium with any system $C$, and $B$ is in equilibrium with $C$ as well, then $A$ is in equilibrium with $B$ [21,22]. We take $C$ here to be a thermometer. More generally, one could assume that $C$ is a system characterized by some intensive quantity $\lambda^{\prime}$ [21,22]. Equilibrium between $A$ and $B$ entails then $\lambda_{A}^{\prime}=\lambda_{B}^{\prime}$ [21,22]. In what follows we concentrate our attention in the Lagrange multiplier associated to the energy. However, the reasoning that follows could be equally applied to any other observable.

Following Gibbs, we make the usual assumptions [2]

1. The interaction energy is negligible

$$
\hat{\mathscr{H}}(A+B) \sim \hat{\mathscr{H}}(A)+\hat{\mathscr{H}}(B) .
$$

2. The subsystems $A$ and $B$ are essentially independent in the sense of the theory of probabilities, i.e.,

$$
\hat{\rho}(A+B) \sim \hat{\rho}(A) \hat{\rho}(B) .
$$

The energy distributions are given here, for each system, by specializing (6) and (7) to the instance $M=1$ and $\hat{O}_{1}^{(G)} \equiv \hat{\mathscr{H}}(G), G=A, B$. It easily follows from Eq. (5) that Ref. [2]

$$
U_{q}(A+B)=U_{q}(A)+U_{q}(B) .
$$

Now, after a bit of algebra Eq. (1) yields (pseudo-additivity [2])

$$
S_{q}(A+B)=S_{q}(A)+S_{q}(B)+(1-q) \frac{S_{q}(A) S_{q}(B)}{k},
$$

which we recast here in the fashion

$$
\begin{aligned}
& \frac{\ln \left[1+\left((1-q) S_{q}(A+B) / k\right)\right]}{1-q} \\
& \quad=\frac{\ln \left[1+\left((1-q) S_{q}(A) / k\right)\right]}{1-q}+\frac{\ln \left[1+\left((1-q) S_{q}(B) / k\right)\right]}{1-q} .
\end{aligned}
$$


We focus our attention now upon Eqs. (21) and (23). For a closed system, both energy and entropy are conserved. As a consequence:

$$
\begin{aligned}
& \delta U_{q}(A)=-\delta U_{q}(B), \\
& \frac{1}{\operatorname{Tr}[\rho(A)]^{q}} \delta\left(\frac{S_{q}(A)}{k}\right)=-\frac{1}{\operatorname{Tr}[\rho(B)]^{q}} \delta\left(\frac{S_{q}(B)}{k}\right) .
\end{aligned}
$$

Introduction of (8) into (25) yields now

$$
\frac{1}{\bar{Z}_{q}(A)^{1-q}} \delta\left(\frac{S_{q}(A)}{k}\right)=-\frac{1}{\bar{Z}_{q}(B)^{1-q}} \delta\left(\frac{S_{q}(B)}{k}\right) .
$$

The next step is to consider the ration between (26) and (24), keeping in mind (13). One immediately finds the equality

$$
\beta^{\prime}(A)=\beta^{\prime}(B) \equiv \beta^{\prime},
$$

i.e., if we set $\beta^{\prime} \propto 1 / T$, thermal equilibrium between $A$ and $B$ arises in a natural fashion and the thermodynamics' zeroth law is obtained. This constitutes the essential result of the present effort. Notice that one assumes here that $\beta^{\prime}$, not $\beta$ (as in Ref. [15]), is proportional to $1 / T$, a fact first observed in Ref. [17] for those special systems whose partition function is of the form $Z_{B G} \propto l^{a}\left(\beta^{\prime}\right)^{-a}$, with $a$ a dimensionless parameter, and $l$ a characteristic length.

In terms of $\beta$ (the TMP Lagrange multiplier) we have (cf. Eq. (13))

$$
\frac{\beta(A)}{\bar{Z}_{q}^{1-q}(A)}=\frac{\beta(B)}{\bar{Z}_{q}^{1-q}(B)} \text {. }
$$

To take $\beta$ as proportional to $1 / T$ forces one to work with a temperature that depends upon the partition function [15].

The present work shows that one can reconcile the zeroth law with Tsallis' thermostatistics without going to the limit $q \rightarrow 1$. In order to assess to what an extent have we succeeded it remains to ascertain the self-consistency of the Gibbs' hypothesis $(19,20)$ within our nonextensive framework. We reconsider the application of Eq. (6) to our present situation and define

$$
\hat{F}(A)=\left[1-(1-q) \beta^{\prime}\left(\hat{\mathscr{H}}(A)-U_{q}(A)\right)\right]^{1 / 1-q},
$$

with a similar expression for $\hat{\mathscr{F}}(B)$. We have then

$$
\hat{\rho}(A) \hat{\rho}(B)=\frac{\hat{F}(A)}{\bar{Z}_{q}(A)} \frac{\hat{F}(B)}{\bar{Z}_{q}(B)},
$$

which, after explicit evaluation, and keeping just first order terms gives

$$
\begin{aligned}
\hat{\rho}(A+B)= & \hat{\rho}(A) \hat{\rho}(B) \\
& -(1-q)\left(\beta^{\prime}\right)^{2} \frac{\left[\hat{\mathscr{H}}(A)-U_{q}(A)\right]}{\bar{Z}_{q}(A)} \frac{\left[\hat{\mathscr{H}}(B)-U_{q}(B)\right]}{\bar{Z}_{q}(B)},
\end{aligned}
$$


which is the promised result. As pointed out in Ref. [24], our subsystems are not exactly independent. But in the last term on the r.h.s. of the above expression is negligible for (i) high temperatures, (ii) the thermodynamic limit (see below), or, of course, for (iii) $q$-values close to unity.

\section{Conclusions}

We have carefully reconsidered the validity of the zeroth law of thermodynamics in a Tsallis' environment. It has been shown to remain approximately valid.

The question revolves around the independence of two independent subsystems and $A, B$ that are brought into thermal contact. We have found that they can be indeed be regarded as independent in quite important instances:

- $q \rightarrow 1$, of course,

- in the high temperature limit, and

- for systems in contact with a heat reservoir, because, if $A$, say, is the reservoir, $\left[\hat{\mathscr{H}}(A)-U_{q}(A)\right]$ is a null operator (the mean energy of a reservoir coincides, by definition, with one of its eigenenergies [21,22]. Now you invoke implicitly the heat reservoir notion whenever you use a thermometer!

\section{Acknowledgements}

The financial support of the National Research Council (CONICET) of Argentina is gratefully acknowledged. F. Pennini acknowledges financial support from UNLP, Argentina.

\section{Appendix. Remarks concerning the first law of thermodynamics}

The problem discussed in the present effort is based on the hypothesis that the first law of thermodynamics holds for Tsallis thermostatistics in general, and for the OLM formalism in particular. This is a reasonable assumption considering that the first law of thermodynamics is nothing but energy conservation. We revisit here, from a Tsallis standpoint, the first law and regard the relation

$$
\mathrm{d} U_{q}=\mathrm{d}^{\prime} Q_{q}+\mathrm{d}^{\prime} W_{q},
$$

as valid, where, obviously, $Q_{q}$ stands for heat, $W_{q}$ for work, and $\mathrm{d}^{\prime}$ represents a non exact differential. We deal with an isolated system composed by two subsystems A and $\mathrm{B}$ that share energy exclusively via heat exchange (see Section 3). As a result $\mathrm{d}^{\prime} W_{q}=0$ and Eq. (A.1) acquires the appearance

$$
\mathrm{d} U_{q}=\mathrm{d}^{\prime} Q_{q} .
$$


Since (i) energy is additive (Eq. (21)) and (ii) the total energy is conserved (Eq. (24)), Eq. (A.2) entails

$$
\mathrm{d}^{\prime} Q_{q}(A)=-\mathrm{d}^{\prime} Q_{q}(B)
$$

as expected. Within Tsallis framework, two systems that interchange energy only via heat transfer verify a heat conservation law. As a bonus, we are straightforwardly led to a possible generalization of the Clausius equation, by asking that appropriate relations for entropy and heat be simultaneously satisfied. We thus propose a generalization of the Clausius equation [21,22]

$$
\mathrm{d} S=\frac{\mathrm{d}^{\prime} Q}{T}
$$

of the form

$$
\mathrm{d} S_{q}=f\left(\bar{Z}_{q}\right) \frac{\mathrm{d}^{\prime} Q}{T}
$$

where $f\left(\bar{Z}_{q}\right)$ is an unknown function to be determined so as to satisfy both Eqs. (25) and (A.3). Introducing Eq. (A.5) into Eq. (25) we obtain

$$
\frac{f\left(\bar{Z}_{q}(A)\right)}{\bar{Z}_{q}^{1-q}(A)} \frac{\mathrm{d}^{\prime} Q(A)}{T}=\frac{f\left(\bar{Z}_{q}(B)\right)}{\bar{Z}_{q}^{1-q}(B)} \frac{\mathrm{d}^{\prime} Q(B)}{T}
$$

that, in view of (A.3), yields

$$
f\left(\bar{Z}_{q}\right)=\bar{Z}_{q}^{1-q}
$$

so that the $q$-generalized Clausius equation can be recast as

$$
\mathrm{d} S_{q}=\frac{\mathrm{d}^{\prime} Q}{T_{q}}
$$

where

$$
T_{q}=\bar{Z}_{q}^{1-q} T=\frac{1}{k \beta}
$$

(Eq. (2) has been used). Special attention must be paid to the fact that $T_{q}$ is not the thermodynamic temperature but a fictitious one, connected to the TMP $\beta$ Lagrange multiplier. It is clear that the traditional equation is recovered in the limit $q \rightarrow 1$. 


\section{References}

[1] C. Tsallis, R.S. Mendes, A.R. Plastino, Physica A 261 (1998) 534.

[2] C. Tsallis, Nonextensive statistical mechanics and thermodynamics: Historical back-ground and present status, in: S. Abe, Y. Okamoto (Eds.), Nonextensive Statistical Mechanics and its Applications, Lecture Notes in Physics, Springer, Berlin, 2001, in press.

[3] A.K. Rajagopal, S. Abe, Phys. Rev. Lett. 83 (1999) 1711.

[4] B.M. Boghosian, Phys. Rev. E 53 (1996) 4754.

[5] S. Abe, A.K. Rajagopal, preprint (2000) [cond-mat/0002159].

[6] D. Vitali, P. Grigolini, Phys. Lett. A 249 (1998) 248.

[7] J. Naudts, preprint (1999) [cond-mat/9904070].

[8] P.T. Landsberg, V. Vedral, Phys. Lett. A 247 (1998) 211.

[9] L.P. Chimento, J. Matt. Phys. 38 (1997) 2565.

[10] C. Tsallis, Braz. J. Phys. 29 (1999) 1.

[11] C. Tsallis, Chaos, Solitons, and Fractals 6 (1995) 539, and references therein; an updated bibliography can be found in http://tsallis.cat.cbpf.br/biblio.htm.

[12] C. Tsallis, Physics World 10 (July 1997) 42.

[13] A.R. Plastino, A. Plastino, in: E. Ludeña (Ed.), Condensed Matter Theories, Vol. 11, Nova Science Publishers, New York, USA, 1996, p. 341.

[14] A.R. Plastino, A. Plastino, Braz. J. of Phys. 29 (1999) 79.

[15] S. Abe, Physica A 269 (1999) 403.

[16] S. Martínez, F. Nicolás, F. Pennini, A. Plastino, Physica A 286 (2000) 489.

[17] S.K. Rama, Phys. Lett. A 276 (2000) 1.

[18] E.T. Jaynes, in: W.K. Ford (Ed.), Statistical Physics, Benjamin, New York, 1963.

[19] A. Katz, Statistical Mechanics, Freeman, San Francisco, 1967.

[20] C. Tsallis, J. Stat. Phys. 52 (1988) 479.

[21] F. Reif, Statistical and Thermal Physics, McGraw-Hill, New York, 1965.

[22] E.A. Desloge, Thermal Physics, Holt, Rinehart and Winston, New York, 1968.

[23] E. Fick, G. Sauerman, The Quantum Statistics of Dynamic Processes, Springer, Berlin, 1990.

[24] G.R. Guerberoff, G.A. Raggio, J. Math. Phys. 37 (1996) 1776. 